\title{
Racial and ethnic disparities in preterm birth outcomes: a call to action for neonatal providers
}

\author{
Heather H. Burris $\mathbb{1}^{1} \cdot$ Margaret G. Parker ${ }^{2}$
}

Received: 22 December 2020 / Revised: 13 January 2021 / Accepted: 14 January 2021 / Published online: 28 January 2021

(c) The Author(s), under exclusive licence to Springer Nature America, Inc. 2021

We read with interest the study by Karvonen et al. entitled "Racial and ethnic disparities in outcomes through 1 year of life in infants born prematurely: a population-based study in California." We commend the authors on pursing this topic using a rich dataset of over 3 million births from 2011 to 2017 that enabled analysis of morbidities of prematurity and covariate adjustment for multiple confounders that are often lacking in other population-based reports. While racism, adverse social determinants of health, and economic disparities in the United States lead to many health inequities, the unequal chance of infant survival is particularly tragic. Non-Hispanic Black infants die at more than twice the rate of White infants (10.8 vs. 4.8 per 1000 live births) [1]. While Hispanic infants die (4.9 per 1000) at similar rates to non-Hispanic White infants, the study by Karvonen et al. highlights that when restricting to preterm infants after discharge, outcomes are less equitable. There were three important findings regarding racial and ethnic disparities among preterm infants in this study that are relevant for neonatal providers and those that care for preterm infants in the first year of life. Compared to non-Hispanic White preterm infants, non-Hispanic Black and Hispanic preterm infants were: (1) less likely to die before discharge, (2) more likely to die after discharge, and (3) more likely to be admitted after discharge.

The first finding, that Black and Hispanic patients are less likely to die in the neonatal intensive care unit (NICU), is particularly important for neonatal providers. At first glance, it might seem like this occurs because of a survival

Heather H. Burris

burrish@chop.edu

1 Division of Neonatology, Department of Pediatrics, Children's Hospital of Philadelphia, University of Pennsylvania Perelman School of Medicine, Philadelphia, PA, USA

2 Department of Pediatrics, Boston Medical Center, Boston University School of Medicine, Boston, MA, USA advantage due to less severe disease among these patients. However, the finding that after discharge these patients are more likely to be readmitted and die argues against illness severity as an explanation. Instead, the finding may reflect decisions regarding timing of death. There is some data to suggest that there are differences in the choice to resuscitate periviable infants at birth. In a study of three states' data (CA, MO, PA) from 1995 to 2005, Black infants born at 23-24 6/7 weeks were more likely to be intubated (resuscitated at birth) [2]. Black race was a significant predictor of intubation after adjustment for other demographic variables and hospital of delivery, suggesting that family preferences may play a role in initiating intensive care. Less is known about the demographics of families choosing to continue intensive care among sick preterm infants in the NICU. In the setting of severe illness and an extremely poor prognosis, palliative care may be an option. It is possible that Black and Hispanic families are less likely to choose palliative care compared to non-Hispanic White families. If so, these choices might reflect varying cultural and religious backgrounds.

An alternative explanation is that interpersonal connections between NICU providers and families differ due to structural factors and implicit biases that impede effective communication. Optimally, the complex decision to redirect care requires understanding of the infant's condition and prognosis in addition to trust between the family and the medical team. If palliative care in the NICU is unequally distributed among families by race/ethnicity, NICU providers have an obligation to ensure this is not due to inability to connect with families from structural factors such as transportation challenges, financial burdens, or language barriers. Furthermore, recognition that implicit biases may also adversely affect connections and communication between NICU providers and families is a necessary step to achieve equity in care and counseling throughout the NICU hospitalization.

If there are differential risk-adjusted death rates by race/ethnicity prior to NICU discharge, then infants may 
differ with respect to their severity of illness at home by race/ethnicity. Only living infants at the time of discharge are eligible for readmissions or death after discharge, which can lead to biases in subsequent analyses; if Black or Hispanic infants are sicker upon NICU discharge from differential uptake of palliative care, then readmissions and death might be less attributable to post-discharge factors. Nonetheless, these undesired outcomes may be partially avoidable and this study challenges us to find prevention strategies.

The findings of increased readmissions and death after discharge among Black and Hispanic infants compared to White infants are consistent with differential mortality rates among infants overall [1]. While these findings were seen after adjustment for infant characteristics, maternal medical conditions, education, insurance status and WIC participation, residual confounding by additional social factors, such as unmet basic needs, likely partially explain these findings [3]. Compared to White families, housing instability and food and energy insecurity are more prevalent among Black and Hispanic families and adversely affect child health [4]. Thus, the American Academy of Pediatrics recommends systematic screening and referral systems for unmet basic needs [5]. While these screenings are increasingly used in the pediatric out-patient setting [6], they are not used routinely in the NICU or in high-risk infant follow-up clinics.

Another important etiology that may explain disparities in readmissions and mortality is access to medical care. Lower attendance in post-discharge subspecialty care, including high-risk clinics dedicated to the needs of preterm infants occurs more often among Black families, compared to White families [7]. Overcoming barriers to clinic participation may include provision of transportation, conducting visits remotely, and/or coordinating home visits. Medical care, however, is just one aspect of an infant's environment. Due to longstanding residential segregation in the U.S., neighborhood conditions differ by race/ethnicity [8]. Black and Hispanic infants are disproportionately exposed to pollution [9] and neighborhood deprivation [10]. Taken together, lack of family and community resources, coupled with environmental toxicant exposures increase risks among Black and Hispanic infants after NICU discharge.

We commend Karvonen et al. for their study, which highlights once again, that when preterm infants are discharged from the NICU, they go out into a world that holds unequal promise of health and longevity by race/ethnicity in America. As a neonatal community, we need to address implicit bias, improve communication with socially disadvantaged families, implement standardized systems for screening and referral for adverse social determinants of health, listen to our families, and advocate for societal change to achieve equity.

\section{Compliance with ethical standards}

Conflict of interest The authors declare that they have no conflict of interest.

Publisher's note Springer Nature remains neutral with regard to jurisdictional claims in published maps and institutional affiliations.

\section{References}

1. Ely DM, Driscoll AK. Infant mortality in the United States, 2018: data from the period linked birth/infant death file. Natl Vital Stat Rep. 2020;69:1-18.

2. Tucker Edmonds B, Fager C, Srinivas S, Lorch S. Racial and ethnic differences in use of intubation for periviable neonates. Pediatrics. 2011;127:e1120-7. https://doi.org/10.1542/peds.20102608.

3. Lorch SA, Enlow E. The role of social determinants in explaining racial/ethnic disparities in perinatal outcomes. Pediatr Res. 2016;79:141-7. https://doi.org/10.1038/pr.2015.199.

4. Braveman P, Egerter S, Williams DR. The social determinants of health: coming of age. Annu Rev Public Health. 2011;32:381-98. https://doi.org/10.1146/annurev-publhealth-031210-101218.

5. Council On Community Pediatrics. Poverty and child health in the United States. Pediatrics. 2016;137. https://doi.org/10.1542/peds. 2016-0339.

6. Sokol R, Austin A, Chandler C, Byrum E, Bousquette J, Lancaster $\mathrm{C}$, et al. Screening children for social determinants of health: a systematic review. Pediatrics. 2019;144. https://doi.org/10.1542/ peds.2019-1622.

7. Swearingen C, Simpson P, Cabacungan E, Cohen S. Social disparities negatively impact neonatal follow-up clinic attendance of premature infants discharged from the neonatal intensive care unit. J Perinatol. 2020;40:790-7. https://doi.org/10.1038/s41372-0200659-4.

8. Nardone A, Casey JA, Morello-Frosch R, Mujahid M, Balmes JR, Thakur N. Associations between historical residential redlining and current age-adjusted rates of emergency department visits due to asthma across eight cities in California: an ecological study. Lancet Planet Health. 2020;4:e24-31. https://doi.org/10.1016/ S2542-5196(19)30241-4.

9. Tessum CW, Apte JS, Goodkind AL, Muller NZ, Mullins KA, Paolella DA, et al. Inequity in consumption of goods and services adds to racial-ethnic disparities in air pollution exposure. Proc Natl Acad Sci USA. 2019;116:6001-6. https://doi.org/10.1073/ pnas. 1818859116

10. Andrews MR, Tamura K, Claudel SE, Xu S, Ceasar JN, Collins BS, et al. Geospatial analysis of neighborhood deprivation index (NDI) for the United States by county. J Maps. 2020;16:101-12. https://doi.org/10.1080/17445647.2020.1750066. 